\title{
Divergência entre genótipos de soja, cultivados em várzea irrigada ${ }^{1}$
}

\author{
Elonha Rodrigues dos Santos ${ }^{2 *}$, Hélio Bandeira Barros ${ }^{3}$, Emerson de Castro Ferraz ${ }^{4}$, Antonio José Silva Cella ${ }^{5}$, \\ Aristóteles Capone ${ }^{6}$, Adão Felipe dos Santos ${ }^{4}$, Rodrigo Ribeiro Fidelis ${ }^{3}$
}

\section{RESUMO}

A divergência genética é um dos mais importantes parâmetros avaliados por melhoristas de plantas, na fase inicial de um programa de melhoramento genético. Diante disso, objetivou-se com este trabalho avaliar, por meio de técnicas multivariadas, a divergência genética entre 48 genótipos de soja, cultivados em várzea irrigada no Estado do Tocantins, com o intuito de identificar as combinações mais promissoras para produzir recombinações superiores, tanto destinados a produção de óleo e farelo, como do grupo especial, destinados ao consumo humano. O experimento foi conduzido no município de Formoso do Araguaia, TO, em cultivo de várzea irrigada na entressafra de 2010. O delineamento experimental foi o de blocos ao acaso, com quatro repetições. Verificou-se variabilidade entre os genótipos testados. Os resultados dos métodos de agrupamento de Tocher, UPGMA e Variáveis Canônicas foram concordantes entre si e detectaram quatro grupos distintos. As seguintes hibridações são promissoras para produção de grãos de soja destinados a óleo e farelo: M-Soy 8766, M-Soy 9144, A 7002 e M-Soy 9056 com Amaralina e cruzamentos entre M-Soy 8766, M-Soy 9144 e Amaralina com BRSMG 790A, BRS 257, BRS 216 e BRS 213 e são indicados visando a genótipos de soja especiais para alimentação humana.

Palavras-chave: Glycine max L., soja tipo alimento, análise multivariada, Tocher, Mahalanobis, UPGMA.

\section{ABSTRACT}

\section{Divergence between soybean genotypes grown in irrigated lowland, Brazil}

The genetic diversity is one of the most important parameters evaluated by plant breeders in the early stages of a genetic improvement program. The objective of this paper was to evaluate the genetic divergence by means of multivariate techniques, among 48 soybean genotypes grown in irrigated lowland in the State of Tocantins, in order to select parents of hybrids for the production of oil and meal, as well as varieties of the panel, intended for human consumption. The experiment was conducted in the county Formoso do Araguaia - Tocantins, Brazil, in the cultivation of irrigated lowland, in the inter-cropping 2010. The experimental design was a randomized block with four replications.

Recebido para publicação em 25/03/2011 e aprovado em 28/11/2011

'Parte da dissertação apresentada ao curso de pós-graduação em Produção Vegetal pela Universidade Federal do Tocantins.

${ }^{2}$ Engenheira-Agrônoma, Doutoranda. Universidade de Brasília, Departamento de Agronomia, Campus Universitário Darcy Ribeiro. Caixa-Postal 4508, 70910-900, Brasília, DF, Brasil. elonharodrigues@yahoo.com.br (*Autor para correspondência.)

${ }^{3}$ Engenheiros-Agrônomos, Doutores. Universidade Federal do Tocantins, Departamento de Fitotecnia, Campus Universitário de Gurupi. Caixa-Postal 66, 77402-970, Gurupi, TO, Brasil.barroshb@pq.cnpq.br; fidelisrr@ @pq.cnpq.br

${ }^{4}$ Graduandos do Curso de Agronomia, Universidade Federal do Tocantins, Departamento de Fitotecnia, Campus Universitário de Gurupi. Caixa-Postal 66, 77402-970, Gurupi, TO, Brasil. emerson-ago@ hotmail.com; adaofelipe@uft.edu.br

${ }^{5}$ Biólogo, Mestrando. Universidade Federal do Tocantins, Departamento de Fitotecnia, Campus Universitário de Gurupi. Caixa-Postal 66, 77402-970, Gurupi, TO, Brasil. cella.antonio86@gmail.com

${ }^{6}$ Engenheiro-Agrônomo, Mestre. Universidade Federal do Tocantins, Campus Universitário de Gurupi. Caixa-Postal 66, 77402-970, Gurupi, TO, Brasil. aristotelescapone @ hotmail.com 
There was observed variability among the genotype tested. The Tocher's method, UPGMA and Canonic Variables agreed among themselves, and found four distinct groups. The following hybrids are promising for the production of soybean oil and meal for the M-Soy 8766, M-Soy 9144, A-7002 and M-soy 9056 with Amaralina RR crosses between and M-Soy 8766, M-Soy 9144 and Amaralina RR with BRSMG 790A, BRS 257, BRS 216 and BRS 213, are listed in order especially soybeans for human consumption.

Key words: Glycine max L., soybean type food, multivariate analysis, Tocher, Mahalanobis, UPGMA.

\section{INTRODUÇÃO}

No ocidente, a soja (Glycine max L.) caracteriza-se, principalmente, como produto para alimentação animal; entretanto, no oriente é, há milênios, cultivada como alimento humano. Os grãos dessa leguminosa são compostos por aproximadamente $40 \%$ de proteínas e $20 \%$ de lipídios. Apresenta ainda em sua constituição açúcares, fibras, carboidratos, além de ser boa fonte de minerais e de vitaminas A, E, B1 e B2 (Bellaver et al., 2002). Nos últimos anos, seus subprodutos ganharam lugar considerável no mercado, por suas propriedades nutracêuticas, com numerosas alegações ligando seus efeitos à promoção da saúde (Duffy et al., 2007).

Atualmente, alguns programas de melhoramento genético vêm desenvolvendo cultivares especiais de soja, do tipo alimento humano, isentos de enzimas lipoxigenases, o que confere melhor sabor aos grãos, a fim de produzir grãos mais aceitáveis pelos consumidores brasileiros (Azevedo et al., 2004 e Sediyama, 2009). Entretanto, para a obtenção de novos cultivares de soja, estudos sobre divergência genética tornam-se necessários, para que se conheça a diversidade existente, tanto de cultivares introduzidos, quanto daqueles adaptados à determinada região.

Segundo Abreu et al. (2004), esse conhecimento sobre a divergência genética, possibilita inferências sobre a capacidade específica de combinação e a heterose, antes de se realizar os cruzamentos, com maior chance de identificar e recuperar combinações mais promissoras nas populações segregantes.

A análise multivariada em estudos sobre divergência genética tem sido uma ferramenta intensivamente utilizada em pesquisas, em várias culturas, como feijão-preto (Elias et al., 2007), trigo (Condé, et al., 2010), milho (Dotto et al., 2010), girassol (Vogt et al., 2010) e soja (Azevedo et al., 2004; Marques, 2010 e Almeida et al., 2011).

Dentre os métodos disponíveis, para estudo da divergência genética, destaca-se o uso da distância generalizada de Mahalanobis, como medida de dissimilaridade, e os métodos de agrupamento de Tocher, de UPGMA e das Variáveis Canônicas. A análise de agrupamento tem por finalidade reunir, por algum critério de classificação, os genitores, em grupos, de tal forma que exista homogeneidade dentro do grupo e heterogeneidade entre grupos (Kloster et al., 2011).

Para Benin et al. (2002), essa estruturação de grupos de divergência é fundamental na escolha de genitores, sendo que cruzamentos a partir de genótipos reunidos em grupos afastados são considerados mais promissores, por serem mais dissimilares.

Estudos realizados por Elias et al., (2007); Vogt et al., (2010), Cabral et al., (2011) e Kloster et al. (2011), sobre a divergência genética em diferentes culturas, obtiveram resultados conclusivos, que permitiram indicar cruzamentos promissores em programas de melhoramento, envolvendo os genótipos testados.

O Estado do Tocantins apresenta área favorável ao cultivo da soja, tanto na safra (terras altas), como no período da entressafra (várzea irrigada). Entretanto, não há cultivares de soja do grupo especial (destinados ao consumo humano) adaptados a esse Estado.

Diante disso, objetivou-se com este trabalho avaliar a divergência genética, por meio de técnicas multivariadas, entre 48 genótipos de soja, cultivados em várzea irrigada no Estado do Tocantins, com o intuito de identificar as combinações mais promissoras para produzir recombinações superiores, tanto destinados à produção de óleo e farelo, como do grupo especial, destinados ao consumo humano.

\section{MATERIAL E MÉTODOS}

O trabalho foi conduzido em várzea irrigada, na entressafra 2010, no município de Formoso do Araguaia, TO, localizado a $11^{\circ} 45^{\prime} \mathrm{S}$ e $49^{\circ} 04^{\prime}$ 'W e $170 \mathrm{~m}$ de altitude, em solo do tipo Gley Pouco-Húmico (Embrapa, 1999). O clima, segundo a classificação de Thornthwaite, é do tipo Aw, úmido com moderada deficiência hídrica, com precipitação anual média de $1.400 \mathrm{~mm}$ e temperatura média anual que varia de 22 a $32{ }^{\circ} \mathrm{C}$ (Seplan, 2003).

O experimento foi implantado no dia 22 de maio de 2010. O delineamento experimental adotado foi o de blo- 
cos casualizados, com 48 tratamentos e quatro repetições. Cada unidade experimental foi composta por quatro linhas de 3,5 m de comprimento, espaçadas entre si por 0,45 m. Os tratamentos foram constituídos por 48 genótipos de soja, pertencentes a diferentes programas de melhoramento (Tabela 1).

Desses, 42 genótipos eram do tipo convencional (destinados à produção de óleo e farelo) e, seis, do grupo especial (destinados à alimentação humana, por apresentarem melhor sabor), sendo BRSMG 800A, AH09-004, BRS 216, BRSMG 790A, BRS 257 e BRS 213 (Tabela 1).

O preparo do solo foi o habitualmente utilizado em várzea irrigada, com aração, gradagem e rolagem (com auxílio de rolo compactador). No momento da semeadura, inocularam-se as sementes com estirpes de Bradyrhizobium japonicum, na dose de $500 \mathrm{~g}$ de inoculante para $500 \mathrm{~kg}$ de sementes, e com fungicida Carbendazim-Thiram 200 SC, na dose de $200 \mathrm{~mL} / 100 \mathrm{~kg}$ de semente. A adubação de semeadura foi realizada com base na análise de solo e nas recomendações para a cultura, correspondendo a $500 \mathrm{~kg}$ $\mathrm{ha}^{-1}$ da formulação NPK 00-16-16 + micronutrientes. A irrigação foi realizada por meio da elevação do lençol freático, de forma a manter o solo com aproximadamente $70 \%$ da capacidade de campo, durante todo o ciclo da cultura (subirrigação). O controle de pragas e doenças foi executado conforme as exigências para campos de produção de sementes.

As seguintes variáveis foram avaliadas: 1) número de dias para o florescimento (NDF), período que correspondeu ao número de dias decorridos entre a emergência das plântulas até $50 \%$ do florescimento das plantas da parcela; 2) número de dias para maturidade (NDM), período que correspondeu ao número de dias decorridos desde a emergência das plântulas, até a data em que $95 \%$ das vagens das plantas da parcela apresentaram-se maduras; 3 ) altura das plantas na maturidade (APM), medições realizadas na fase de maturação, medindo-se da base da planta até a inserção do racimo no ápice da haste principal, em cinco plantas da área útil; 4) altura de inserção da primeira vagem (AIV), medições realizadas na fase de maturação, medindo-se a distância, a partir da superfície do solo, até a primeira vagem da haste principal, em cinco plantas da área útil; 5) clorofila, cujos dados foram obtidos por leituras diretas no estádio $\mathrm{R}_{6}$, coletando-se nove amostras por parcela, com uso de um clorofilômetro da marca ClorofiLOG® modelo CFL 1030, produzido pela Falker Automação Agrícola. Os valores dos índices de clorofila foram expressos em ICF (Índice de Clorofila Falker), sendo determinados os valores de clorofila a, b e total; 6) número de sementes por vagem (NSV), obtido da média de cinco plantas por contagem direta das sementes por vagem; 7) número de sementes por plantas (NSP), média obtida pela contagem direta de sementes de cinco plantas; 8) número de vagens por plantas (NVP), média obtida pela contagem direta de vagens de cinco plantas; 9) percentagem de vagens chochas (VC), valores médios obtidos pela contagem direta de todas as vagens de cinco plantas, posteriormente, transformados em percentagem de vagens chochas; 10) percentagem de vagens com uma semente (V1S), valores médios obtidos pela contagem direta de todas as vagens de cinco plantas, posteriormente, transformados em percentagem de vagens com uma semente; 11) percentagem de vagens com duas sementes (V2S), valores médios obtidos pela contagem direta de todas as vagens de cinco plantas, posteriormente, transformados em percentagem de vagens com duas sementes; 12 ) percentagem de vagens com três sementes (V3S), valores médios obtidos pela contagem direta de todas as vagens de cinco plantas, posteriormente, transformados em percentagem de vagens com três sementes; 13) massa de 100 sementes (MCS), obtida de uma amostra de 100 sementes; 14) peso hectolitro (PH), obtido de uma amostra $1000 \mathrm{~mL}$ de sementes; 15) rendimento de sementes (RS), determinado após a trilhagem das plantas e limpeza das sementes, com posterior correção da umidade dos grãos para $13 \%$.

Os dados foram submetidos à análise de variância e ao teste de F. Quando constatado efeito significativo de

Tabela 1. Genótipos de soja, utilizados no experimento

\begin{tabular}{lllll}
\hline 1. BRS Luziânia & 11. AH09-002 & 21. NK 9074 RR & 31. BRS Valiosa RR & 41. M 8585 RR \\
2. BRSMG 800A & 12. Anta 83 RR & 22. CD 240 RR & 32. TMG1182 RR & 42. M 8866 RR \\
3. AH09-004 & 13. Anta 82 RR & 23. COCKER 16 & 33. GB 874 & 43. AN 8500 \\
4. BRS 216 & 14. M-Soy 9056 & 24. AH09-024 & 34. TMG155 RR & 44. A 7002 \\
5. AH09-003 & 15. BRS8560 RR & 25. P98Y70 RR & 35. M 8925 RR & 45. STS 750 \\
6. BRSMG 790A & 16. Juliana & 26.Amaralina & 36. TMG1181 RR & 46. TMG7188 RR \\
7. AH09-001 & 17. P98Y51 RR & 27. M-Soy7211 & 37. M 8787 RR & 47. M 8527 RR \\
8. BRS 257 & 18. Juliana RR & 28. NK 7074 RR & 38. TMG132 RR & 48. GB 881 \\
9. BRSGO 8660 & 19. CD 219 RR & 29. M-Soy 8766 & 39. M 8867 RR & \\
10. BRS 213 & 20. M 9144 RR & 30. P 99R01 RR & 40. TMG1187 RR & \\
\hline
\end{tabular}

Genótipos especiais (destinados à alimentação humana): 2-BRSMG 800A, 3-AH09-004, 4-BRS 216,

6-BRSMG 790A, 8-BRS 257 e 10-BRS 213. 
tratamentos, as médias foram agrupadas pelo teste de Scott-Knott, a 5\% de probabilidade. A distância genética entre todos os pares de genótipos foi estimada por meio da distância generalizada de Mahalanobis e pelo método de análise de variáveis canônicas. Com base na distância generalizada de Mahalanobis, aplicaram-se os métodos de agrupamentos aglomerativos de Tocher e UPGMA, com auxílio do programa computacional Genes (Cruz, 2008).

\section{RESULTADOS E DISCUSSÃO}

A análise de variância mostrou diferença significativa entre os tratamentos, para todas as características, exceto para clorofila a (Tabela 2). Estes resultados demonstram a existência de variabilidade entre os genótipos, possibilitando, assim, a obtenção de ganhos genéticos em programas de melhoramento, com o uso desses genótipos como progenitores.

Os resultados médios das variáveis estudadas nos 48 genótipos de soja encontram-se na Tabela 3 . O número de dias para o florescimento foi a característica que possibilitou maior discriminação entre os tratamentos, ou seja, aquela em que se obteve o maior número de grupos significativamente diferentes. Os valores para essa característica oscilaram entre 24 (BRS 216) e 48 (Amaralina) dias, evidenciando a variabilidade entre os genótipos analisados.

O número de dias para a maturação variou de 75 a 130 dias, ciclos classificados como precoce e médio, segundo Sediyama (2009). O grupo mais precoce foi composto pelos genótipos BRS 257, BRS 213, Anta 82 RR, BRS 216 e Anta 83 RR, que completaram o ciclo em média aos 77
DAE, comprovando assim, a precocidade desses cultivares na região de estudo. $\mathrm{O}$ fator que pode ter influenciado no ciclo desses cultivares foi a sensibilidade fotoperiódica, uma vez que esses genótipos foram lançados em regiões de alta latitude, principalmente genótipos tipo alimento, como BRS 257, BRS 213 e BRS 216, que são recomendados para a região sul do país (Embrapa, 2008). O Amaralina apresentou um ciclo de 130 dias, sendo, portanto, a mais tardia.

A altura média de plantas oscilou de 19,10 a 64,15 cm. $\mathrm{O}$ grupo que apresentou menor estatura foi composto por BRS 257 e BRS 216 (média de $20 \mathrm{~cm}$ ) e, o grupo integrado pelos genótipos AH09-001 e Amaralina, a maior estatura. O genótipo Amaralina apresentou ainda, maior altura de inserção de vagem, diferindo significativamente dos demais.

Os valores de clorofilas a, b e total encontram-se na Tabela 3. Para clorofila a, não foi verificada diferença significativa entre os genótipos. Para clorofila b e clorofila total, constatou-se a formação de dois grupos distintos. Independentemente dos tratamentos, verificou-se que os valores de clorofila a foram superiores aos de clorofila $b$. Segundo Critchley (1999), isto se deve à maior proporção do fotossistema I, que é mais rico em clorofila a, e está diretamente relacionado com a capacidade das plantas em maximizar a captura de luz em condições normais de luminosidade.

Em média, cada vagem produziu 2,14 sementes (NSV) (Tabela 3). Esses resultados assemelharam-se aos obtidos por Almeida et al. (2011), em cultivares de soja culti-

Tabela 2. Resumo da análise de variância de 48 genótipos de soja, cultivados em várzea irrigada, em Formoso do Araguaia, TO, na entressafra de 2010, NDF: número de dias para o florescimento; NDM: número de dias para a maturação; AIV: altura da inserção da primeira vagem; AP: altura das plantas; CLa: clorofila a; CLb: clorofila b; CLtotal: clorofila total; NSV: número de sementes por vagem; NSP: número de sementes por plantas; NVP: número de vagens por plantas; VC: percentagem de vagens chochas; V1S: percentagem de vagens com uma semente; V2S: percentagem de vagens com duas sementes; V3S: percentagem de vagens com três sementes; MCS: massa de 100 sementes; PH: peso de hectolitro e RS: rendimento de sementes por hectare

\begin{tabular}{|c|c|c|c|c|c|c|c|c|c|c|}
\hline \multirow{2}{*}{ FV } & \multirow{2}{*}{ GL } & \multicolumn{9}{|c|}{ Quadrado médio } \\
\hline & & NDF & NDM & AP & AIV & CLa & CLb & CLtotal & NSV & NSP \\
\hline Blocos & 3 & 1,6 & 11,1 & 39,7 & 16,5 & 2,6 & 1,3 & 1,5 & 0,005 & 4310 \\
\hline Tratamentos & 47 & $136,1 * *$ & $399,2 * *$ & $329,7 * *$ & $14,4 * *$ & $1,1^{\mathrm{ns}}$ & $2,0 * *$ & $3,9^{*}$ & $0,130 * *$ & $12145^{* *}$ \\
\hline Resíduos & 141 & 0,5 & 7,6 & 11,6 & 2,1 & 1,1 & 0,81 & 2,4 & 0,012 & 1108 \\
\hline Média & & 33,84 & 94,86 & 37,66 & 7,76 & 34,33 & 12,54 & 46,87 & 2,14 & 155,52 \\
\hline $\mathrm{CV}(\%)$ & & 2,14 & 2,91 & 9,06 & 18,60 & 3,02 & 7,18 & 3,32 & 5,29 & 21,41 \\
\hline \multirow{2}{*}{ FV } & \multirow{2}{*}{ GL } & \multicolumn{9}{|c|}{ Quadrado médio } \\
\hline & & NVP & $\mathrm{VC}$ & V1S & & & V3S & MCS & $\mathbf{P H}$ & $\mathbf{R S}$ \\
\hline Blocos & 3 & 853 & 1,4 & 1,2 & & 3,6 & 16,0 & 0,3 & 0,0001 & 543582 \\
\hline Tratamentos & 47 & $2261 * *$ & $45,6^{* *}$ & $228,4 * *$ & & $5 * *$ & $519,9 * *$ & $15,8^{* *}$ & $0,0023 * *$ & $543173 * *$ \\
\hline Resíduos & 141 & 215 & 1,4 & 15,6 & &, 9 & 31,5 & 0,2 & 0,0001 & 466670 \\
\hline Média & & 71,80 & 3,89 & 13,76 & & & 34,47 & 16,21 & 75,1 & 2729 \\
\hline $\mathrm{CV}(\%)$ & & 20,41 & 30,77 & 28,68 & & & 16,29 & 2,70 & 1,56 & 25,03 \\
\hline
\end{tabular}

** e $*$ significativos a $\mathrm{P} \leq 0,01$ e $\mathrm{P} \leq 0,05$, respectivamente, pelo teste $\mathrm{F}$, ${ }^{\text {ns }}$ não significativo, pelo teste $\mathrm{F}$.

Rev. Ceres, Viçosa, v. 58, n.6, p. 755-764, nov/dez, 2011 
vados na entressafra de 2005, em Formoso do Araguaia, com média de 2,13 sementes por vagens.

Ainda, na Tabela 3, observa-se que houve diferença significativa no número de sementes por planta (NSP). O grupo inferior foi composto pelos genótipos BRS 257, BRS 213, AH09-004. Em contraste, os genótipos P98Y70 RR, M 8925 RR, M-Soy 9144, P98Y51 RR, BRS Valiosa RR, TMG 132 RR e M 8867 RR compuseram o grupo com maior NSP. Constatou-se, ainda, que a maioria dos genótipos tipo alimento incluía-se nos grupos que apresentaram menores NSP, um indicativo da sua falta de adaptação às condições de cultivo.

Foram obtidos cinco grupos distintos para número de vagens por planta (NVP). Os genótipos M-8867 RR e TMG 132 RR integraram o grupo que apresentou maiores valores para NVP, com média de 127 vagens por planta; os genótipos BRS 257, BRS 213 e AH09-004 formaram o grupo inferior para MVP, com média de 28 vagens por planta, oscilação de $78 \%$ para essa característica, o que demonstra a variabilidade entre os genótipos estudados.

O genótipo Anta $82 \mathrm{RR}$ apresentou $23 \%$ de vagens chochas (Tabela 3), diferindo significativamente dos demais. Esse percentual elevado, possivelmente, ocorreu pelo número elevado de vagens emitidas por planta (característica genética), aliado a ausência de adaptação do cultivar ao local de cultivo (ciclo completo em 77 dias). Assim, essas plantas não possuíam reserva de fotoassimilados suficiente para serem translocados aos grãos, resultando esse elevado percentual de chochamento.

Para percentagem de vagens com uma semente (V1S) (Tabela 3), o genótipo AH09-004 apresentou os maiores valores, com 49\%, diferindo dos demais, e AH09-024 teve o menor percentual, com apenas $5 \%$. Observou-se, ainda, que essa característica, possivelmente tenha relação direta com rendimento de sementes, uma vez que o genótipo AH09-004 foi dos que apresentaram menor rendimento.

O genótipo Juliana forneceu o maior número de vagens com duas sementes (V2S), sendo 69\% superior ao dos demais. O grupo composto pelos genótipos M 8925 RR, TMG 1181 RR, NK 7074 RR, AN 8500, AH09-003, BRS Valiosa RR, Anta 82 RR, CD 219 RR, TNG 155 RR e AH09024 apresentou menor percentual de V2S (média de 36\%), $33 \%$ inferior ao do cultivar Juliana.

O grupo integrado pelos genótipos AH09-024 e CD 219 RR apresentou maior percentual de vagens com três sementes (V3S), com média de 62\%. Em contraste, AH09004 obteve apenas 4\% de V3S, esse menor percentual, provavelmente, podendo ser atribuído à maior proporção de vagens com uma semente.

Ainda na Tabela 3, observa-se que os genótipos tipo alimento (BRSMG 800A, AH09-004, BRS 216, BRSMG 790A, BRS 257 e BRS 213) apresentaram percentuais médios de 4; 27; 46 e 23\% de vagens chochas, com uma semente, com duas sementes e com três sementes, respectivamente.

A massa de 100 sementes formou dez grupos significativamente distintos e os valores variaram de 12,44 a $20,19 \mathrm{~g}$. Os grupos mais contrastantes foram formados pelos genótipos TMG 7188 RR, BRS MG 800A e AH09004, com média de 20,12 g, e pelo grupo dos genótipos COCKER 16 e BRS 216, com média de 12,67 g. A massa de 100 sementes é característica importante na escolha do cultivar, pois a aquisição de sementes de menor massa resultará em menor custo de produção.

Por outro lado, sementes de grãos maiores são preferidas para os cultivares tipo alimento, principalmente para a fabricação de tofu e extrato hidrossolúvel de soja (leite de soja), enquanto cultivares com grãos menores são indicados para a produção de natto (alimento fermentado) (Sediyama, 2009).

A média geral para o peso de hectolitro foi de $75,1 \mathrm{~kg}$ $\mathrm{hL}^{-1}$, com amplitude de $11,0 \mathrm{~kg} \mathrm{hL}^{-1}$. O peso de hectolitro é entendido como densidade das sementes (massa/volume), sendo, ocasionalmente, muito útil quanto à soja destinada à exportação em navios graneleiros, que usam o montante em volume e nem sempre em toneladas de grãos (Marques, 2010).

O rendimento médio de sementes foi de $2729 \mathrm{~kg} \mathrm{ha}^{-1}$. Os genótipos que apresentaram maiores rendimentos são todos recomendados para cultivo no Estado do Tocantins. Dos genótipos tipo alimento, observou-se rendimento médio de $1418 \mathrm{~kg} \mathrm{ha}^{-1}$. Destes, o BRSMG 790A sobressaiu-se aos demais, com produção média de $2387 \mathrm{~kg} \mathrm{ha}^{-1}$. Esse fato confirma a perspectiva de sua utilização no melhoramento de soja tipo alimento para a região do Tocantins, pois permitirá obter maior ganho de seleção, principalmente para produção de grãos.

Os valores para rendimento de grãos foram, em sua maioria, superiores aos observados por Peluzio et al. (2010), no estudo de adaptabilidade e estabilidade de soja na entressafra de 2006 em várzea irrigada em Formoso do Araguaia, TO, em que obtiveram média de $1142 \mathrm{~kg} \mathrm{ha}^{-1}$, e assemelhou-se ao rendimento médio de sementes obtidos por Almeida et al (2011), na entressafra de 2005, em várzea irrigada, em que obtiveram média geral de $2656 \mathrm{~kg}$ $\mathrm{ha}^{-1}$, sendo que os cultivares mais adaptados apresentaram médias superiores a $3000 \mathrm{~kg} \mathrm{ha}^{-1}$.

As contribuições relativas de cada característica na dissimilaridade genética, segundo método de Singh (1981), encontram-se na Tabela 4. As características que mais contribuíram para essa dissimilaridade foram: percentagem de vagens com três sementes $(32,62 \%)$, percentagem de vagens com uma semente $(23,16 \%)$, florescimento $(13,42 \%)$ e percentagem de vagens chochas $(8,23 \%)$, sendo as mais eficientes em explicar a dissimilaridade entre os genótipos testados. No estudo sobre divergência ge- 
Tabela 3. Médias de 17 características avaliadas em 48 genótipos de soja, e grupos de genótipos estabelecidos pelo método de otimização Tocher, com uso da distância generalizada de Mahalanobis NDF: número de dias para o florescimento; NDM: número de dias para a maturação; AIV: altura da inserção da primeira vagem; AP: altura das plantas; CLa: clorofila a; CLb: clorofila b; CLtotal: clorofila total; NSV: número de sementes por vagem; NSP: número de sementes por plantas; NVP: número de vagens por plantas; VC: percentagem de vagens chochas; V1S: percentagem de vagens com uma semente; V2S: percentagem de vagens com duas sementes; V3S: percentagem de vagens com três sementes; MCS: massa de 100 sementes; PH: peso de hectolitro e RS: rendimento de sementes por hectare

\begin{tabular}{|c|c|c|c|c|c|c|c|c|c|c|c|c|c|c|c|c|c|c|}
\hline Genótipos & NDF & NDM & $\begin{array}{c}\mathbf{A P} \\
(\mathbf{c m})\end{array}$ & $\begin{array}{l}\text { AIV } \\
(\mathbf{c m})\end{array}$ & CLa & CLb & CLtotal & NSV & NSP & NVP & $\begin{array}{l}\mathrm{VC} \\
(\%) \\
\end{array}$ & $\begin{array}{l}\text { V1S } \\
(\%) \\
\end{array}$ & $\begin{array}{l}\text { V2S } \\
(\%) \\
\end{array}$ & $\begin{array}{l}\text { V3S } \\
(\%) \\
\end{array}$ & $\begin{array}{c}\text { MCS } \\
(\mathrm{g}) \\
\end{array}$ & $\begin{array}{c}\text { PH } \\
\left(\mathrm{kg} \mathrm{hL}^{-1}\right)\end{array}$ & $\begin{array}{c}\text { RS } \\
\left(\mathrm{kg} \mathrm{ha}^{-1}\right) \\
\end{array}$ & Tocher \\
\hline M-Soy 8766 & $36 \mathrm{~g}$ & $100 \mathrm{c}$ & $35,50 \mathrm{~d}$ & $8,95 \mathrm{c}$ & 33,97 a & 12,95 a & $46,92 \mathrm{a}$ & $2,08 \mathrm{c}$ & $154,80 \mathrm{c}$ & $74,50 \mathrm{c}$ & $2,47 \mathrm{~d}$ & $12,87 \mathrm{~d}$ & $59,05 \mathrm{~b}$ & $25,60 \mathrm{~d}$ & $14,58 \mathrm{~h}$ & $73,5 \mathrm{f}$ & $4572 \mathrm{a}$ & 1 \\
\hline M-Soy 9144 & $40 \mathrm{c}$ & $95 \mathrm{~d}$ & $47,45 \mathrm{~b}$ & $10,65 \mathrm{~b}$ & 35,07 a & $2,17 \mathrm{~b}$ & $47,27 \mathrm{a}$ & $2,25 \mathrm{~b}$ & $227,50 \mathrm{a}$ & $101,05 \mathrm{~b}$ & $3,00 \mathrm{~d}$ & $7,15 \mathrm{e}$ & $51,12 \mathrm{c}$ & $38,77 \mathrm{c}$ & $16,31 \mathrm{f}$ & $74,7 \mathrm{e}$ & $4202 \mathrm{a}$ & 1 \\
\hline A 7002 & $39 \mathrm{~d}$ & $107 \mathrm{~b}$ & $47,25 \mathrm{~b}$ & $8,65 \mathrm{c}$ & $34,75 \mathrm{a}$ & $11,85 \mathrm{~b}$ & $46,60 \mathrm{~b}$ & $2,26 \mathrm{~b}$ & $166,95 \mathrm{c}$ & $73,75 \mathrm{c}$ & $1,27 \mathrm{e}$ & $8,10 \mathrm{e}$ & $53,85 \mathrm{c}$ & $36,75 \mathrm{c}$ & $14,73 \mathrm{~h}$ & $72,2 \mathrm{f}$ & 3957 a & 1 \\
\hline M-Soy 9056 & $40 \mathrm{c}$ & $110 \mathrm{~b}$ & $50,62 \mathrm{~b}$ & $10,67 \mathrm{~b}$ & $35,25 \mathrm{a}$ & $13,62 \mathrm{a}$ & $48,87 \mathrm{a}$ & $2,22 \mathrm{~b}$ & $117,70 \mathrm{c}$ & $52,95 \mathrm{~d}$ & $1,40 \mathrm{e}$ & $9,02 \mathrm{e}$ & $56,40 \mathrm{~b}$ & $33,15 \mathrm{c}$ & $16,10 \mathrm{f}$ & $75,0 \mathrm{e}$ & 3957 a & 1 \\
\hline M 8866 RR & $36 \mathrm{~g}$ & $100 \mathrm{c}$ & $32,00 \mathrm{e}$ & $6,75 \mathrm{~d}$ & 33,77 a & $12,70 \mathrm{a}$ & $46,45 \mathrm{~b}$ & $1,97 \mathrm{~d}$ & $118,92 \mathrm{c}$ & $60,32 \mathrm{c}$ & $3,07 \mathrm{~d}$ & $18,50 \mathrm{c}$ & $56,92 \mathrm{~b}$ & $21,52 \mathrm{~d}$ & $16,68 \mathrm{e}$ & $74,0 \mathrm{e}$ & $3885 \mathrm{a}$ & 1 \\
\hline Juliana & $37 \mathrm{f}$ & $100 \mathrm{c}$ & $35,15 \mathrm{~d}$ & $8,65 \mathrm{c}$ & $34,22 \mathrm{a}$ & $13,50 \mathrm{a}$ & $47,72 \mathrm{a}$ & $2,04 \mathrm{c}$ & $139,60 \mathrm{c}$ & $68,40 \mathrm{c}$ & $1,60 \mathrm{e}$ & $10,90 \mathrm{e}$ & $69,42 \mathrm{a}$ & $18,10 \mathrm{e}$ & $16,18 \mathrm{f}$ & $73,7 \mathrm{f}$ & $3830 \mathrm{a}$ & 1 \\
\hline STS 750 & $37 \mathrm{f}$ & $100 \mathrm{c}$ & $38,40 \mathrm{c}$ & $6,35 \mathrm{~d}$ & 33,95 a & $12,97 \mathrm{a}$ & $46,92 \mathrm{a}$ & $1,96 \mathrm{~d}$ & $120,50 \mathrm{c}$ & $60,85 \mathrm{c}$ & $2,10 \mathrm{~d}$ & $19,95 \mathrm{c}$ & $57,72 \mathrm{~b}$ & $20,22 \mathrm{~d}$ & $17,99 \mathrm{c}$ & $74,7 \mathrm{e}$ & $3765 \mathrm{a}$ & 1 \\
\hline P98Y70 RR & $44 \mathrm{~b}$ & $98 \mathrm{c}$ & $42,25 \mathrm{c}$ & $8,80 \mathrm{c}$ & $34,65 \mathrm{a}$ & $12,87 \mathrm{a}$ & $47,52 \mathrm{a}$ & $2,33 \mathrm{~b}$ & $212,90 \mathrm{a}$ & $96,07 \mathrm{~b}$ & $1,50 \mathrm{e}$ & 6,15 e & $43,20 \mathrm{~d}$ & $49,15 \mathrm{~b}$ & $16,79 \mathrm{e}$ & $75,2 \mathrm{e}$ & $3765 \mathrm{a}$ & 1 \\
\hline M 8527 RR & $36 \mathrm{~g}$ & $107 \mathrm{~b}$ & $35,75 \mathrm{~d}$ & $7,85 \mathrm{c}$ & $34,30 \mathrm{a}$ & $13,00 \mathrm{a}$ & $47,32 \mathrm{a}$ & $2,08 \mathrm{c}$ & $195,35 \mathrm{~b}$ & $93,15 \mathrm{~b}$ & $2,30 \mathrm{~d}$ & $16,12 \mathrm{~d}$ & $54,15 \mathrm{c}$ & $27,42 \mathrm{~d}$ & $19,10 \mathrm{~b}$ & $76,0 \mathrm{~d}$ & $3715 \mathrm{a}$ & 1 \\
\hline GB 881 & $37 \mathrm{f}$ & $100 \mathrm{c}$ & $42,40 \mathrm{c}$ & $9,35 \mathrm{~b}$ & 34,25 a & $13,42 \mathrm{a}$ & $47,70 \mathrm{a}$ & $2,21 \mathrm{~b}$ & $197,67 \mathrm{~b}$ & $89,27 \mathrm{~b}$ & $1,87 \mathrm{e}$ & $10,22 \mathrm{e}$ & $53,07 \mathrm{c}$ & $34,82 \mathrm{c}$ & $19,21 \mathrm{~b}$ & $74,2 \mathrm{e}$ & $3686 a$ & 1 \\
\hline AH09-024 & $37 \mathrm{f}$ & $100 \mathrm{c}$ & $36,25 \mathrm{~d}$ & $8,70 \mathrm{c}$ & 34,30 a & 13,27 a & $47,52 \mathrm{a}$ & $2,56 \mathrm{a}$ & $144,30 \mathrm{c}$ & $56,30 \mathrm{~d}$ & $0,47 \mathrm{e}$ & $5,00 \mathrm{e}$ & $31,22 \mathrm{e}$ & $62,70 \mathrm{a}$ & $16,23 \mathrm{f}$ & $74,0 \mathrm{e}$ & $3663 \mathrm{a}$ & 1 \\
\hline CD 219 RR & $39 \mathrm{~d}$ & $100 \mathrm{c}$ & $36,65 \mathrm{c}$ & $9,40 \mathrm{~b}$ & 34,92 a & $13,55 \mathrm{a}$ & $48,50 \mathrm{a}$ & $2,54 \mathrm{a}$ & $126,00 \mathrm{c}$ & $49,40 \mathrm{~d}$ & $2,42 \mathrm{~d}$ & $6,05 \mathrm{e}$ & $31,50 \mathrm{e}$ & $61,77 \mathrm{a}$ & $15,90 \mathrm{f}$ & $74,7 \mathrm{e}$ & $3631 \mathrm{a}$ & 1 \\
\hline M 8787 RR & $38 \mathrm{e}$ & 90 e & $40,85 \mathrm{c}$ & $9,75 \mathrm{~b}$ & $35,12 \mathrm{a}$ & $11,82 \mathrm{~b}$ & $46,95 \mathrm{a}$ & $2,16 \mathrm{c}$ & $203,05 b$ & $93,70 \mathrm{~b}$ & $3,30 \mathrm{~d}$ & $13,72 \mathrm{~d}$ & $47,47 \mathrm{~d}$ & $35,50 \mathrm{c}$ & $17,37 \mathrm{~d}$ & $72,7 \mathrm{f}$ & $3623 \mathrm{a}$ & 1 \\
\hline BRS 8560 RR & $36 \mathrm{f}$ & $100 \mathrm{c}$ & $33,60 \mathrm{~d}$ & $8,20 \mathrm{c}$ & 34,32 a & $12,67 \mathrm{a}$ & 46,9 & $2,27 \mathrm{~b}$ & $163,20 \mathrm{c}$ & $68,75 \mathrm{c}$ & $3,92 \mathrm{~d}$ & $10,42 \mathrm{e}$ & $44,70 \mathrm{~d}$ & $40,95 \mathrm{c}$ & $16,31 \mathrm{f}$ & $74,7 \mathrm{e}$ & 355 & 1 \\
\hline AN 8500 & $38 \mathrm{~g}$ & $99 \mathrm{c}$ & $49,25 \mathrm{~b}$ & $10,10 \mathrm{~b}$ & 34,20 a & $12,02 \mathrm{~b}$ & $46,20 \mathrm{~b}$ & $2,47 \mathrm{a}$ & $162,55 \mathrm{c}$ & $65,40 \mathrm{c}$ & $0,17 \mathrm{e}$ & $7,75 \mathrm{e}$ & $37,07 \mathrm{e}$ & $55,00 \mathrm{~b}$ & $13,52 \mathrm{i}$ & $73,5 \mathrm{f}$ & 3523 a & 1 \\
\hline M 8867 RR & $38 \mathrm{e}$ & $99 \mathrm{c}$ & $36,50 \mathrm{~d}$ & $8,25 \mathrm{c}$ & $33,52 \mathrm{a}$ & $12,47 \mathrm{a}$ & $46,00 \mathrm{~b}$ & $2,20 \mathrm{~b}$ & $286,20 \mathrm{a}$ & $128,40 \mathrm{a}$ & $4,97 \mathrm{c}$ & $8,60 \mathrm{e}$ & $47,72 \mathrm{~d}$ & $38,75 \mathrm{c}$ & $14,52 \mathrm{~h}$ & $75,0 \mathrm{e}$ & 3519 a & 1 \\
\hline Juliana RR & $36 \mathrm{~g}$ & $100 \mathrm{c}$ & $36,65 \mathrm{~d}$ & $9,75 \mathrm{~b}$ & 33,70 a & $12,57 \mathrm{a}$ & & & $126,45 \mathrm{c}$ & $60,22 \mathrm{c}$ & $2,15 \mathrm{~d}$ & $10,45 \mathrm{e}$ & $63,80 \mathrm{~b}$ & $23,60 \mathrm{~d}$ & $16,20 \mathrm{f}$ & $74,2 \mathrm{e}$ & 344 & 1 \\
\hline TMG 1182 RR & $40 \mathrm{c}$ & $100 \mathrm{c}$ & $38,60 \mathrm{c}$ & $8,95 \mathrm{c}$ & 33,87 a & $12,45 \mathrm{a}$ & $46,30 \mathrm{~b}$ & $2,15 \mathrm{c}$ & $196,67 b$ & $90,87 \mathrm{~b}$ & $5,65 \mathrm{~b}$ & $10,20 \mathrm{e}$ & $47,17 \mathrm{~d}$ & $37,02 \mathrm{c}$ & $18,51 \mathrm{c}$ & $75,0 \mathrm{e}$ & 305 & 1 \\
\hline M 8585 RR & $39 \mathrm{~d}$ & $99 \mathrm{c}$ & $31,35 \mathrm{e}$ & $6,55 \mathrm{~d}$ & $34,05 \mathrm{a}$ & $13,72 \mathrm{a}$ & $47,77 \mathrm{a}$ & $2,10 \mathrm{c}$ & $206,10 b$ & $97,20 \mathrm{~b}$ & $1,27 \mathrm{e}$ & $14,80 \mathrm{~d}$ & $56,75 \mathrm{~b}$ & $27,17 \mathrm{~d}$ & $18,23 \mathrm{c}$ & $73,2 \mathrm{f}$ & 2944 b & 1 \\
\hline & $37 \mathrm{~g}$ & & & & & & & & & & & & & & & & & 1 \\
\hline TMG 7188 RR & $39 \mathrm{~d}$ & $110 \mathrm{~b}$ & 31,70 e & $6,30 \mathrm{~d}$ & 33,95 a & $12,25 \mathrm{~b}$ & $46,22 \mathrm{~b}$ & $2,08 \mathrm{c}$ & $173,55 \mathrm{c}$ & $83,20 \mathrm{c}$ & $4,90 \mathrm{c}$ & $11,25 \mathrm{~d}$ & $55,10 \mathrm{c}$ & $28,72 \mathrm{~d}$ & 20,19 a & $74,2 \mathrm{e}$ & $2831 \mathrm{~b}$ & 1 \\
\hline GB 874 & $36 \mathrm{f}$ & $91 \mathrm{e}$ & $41,80 \mathrm{c}$ & $8,60 \mathrm{c}$ & $33,22 \mathrm{a}$ & $11,77 \mathrm{~b}$ & $45,00 \mathrm{~b}$ & $2,10 \mathrm{c}$ & $136,02 \mathrm{c}$ & $64,77 \mathrm{c}$ & $2,70 \mathrm{~d}$ & $10,80 \mathrm{e}$ & $60,20 \mathrm{~b}$ & $26,32 \mathrm{~d}$ & $17,56 \mathrm{~d}$ & $75,0 \mathrm{e}$ & $2746 b$ & 1 \\
\hline M 8925 RR & $38 \mathrm{e}$ & $100 \mathrm{c}$ & & & & $12,67 \mathrm{a}$ & $46,60 \mathrm{~b}$ & $2,22 \mathrm{~b}$ & 224,85 a & $101,10 \mathrm{~b}$ & $6,50 \mathrm{~b}$ & $9,55 \mathrm{e}$ & $39,75 \mathrm{e}$ & $44,15 \mathrm{c}$ & $13,61 \mathrm{i}$ & $72,7 \mathrm{f}$ & $2674 \mathrm{~b}$ & 1 \\
\hline TMG 1187 RR & $38 \mathrm{e}$ & $100 \mathrm{c}$ & $34,00 \mathrm{~d}$ & $7,35 \mathrm{c}$ & 34,87 a & $12,47 \mathrm{a}$ & $47,35 \mathrm{a}$ & $2,27 \mathrm{~b}$ & $189,15 \mathrm{~b}$ & $83,45 \mathrm{c}$ & $4,52 \mathrm{c}$ & 7,32 e & $44,07 \mathrm{~d}$ & $44,07 \mathrm{c}$ & $14,49 \mathrm{~h}$ & $73,5 \mathrm{f}$ & $2439 \mathrm{~b}$ & 1 \\
\hline P98Y51 RR & $37 \mathrm{f}$ & $100 \mathrm{c}$ & $30,60 \mathrm{e}$ & $6,95 \mathrm{~d}$ & 34,82 a & 12,97 a & $47,82 \mathrm{a}$ & $2,12 \mathrm{c}$ & $235,90 \mathrm{a}$ & $110,40 \mathrm{~b}$ & $2,60 \mathrm{~d}$ & $14,20 \mathrm{~d}$ & $51,82 \mathrm{c}$ & $31,40 \mathrm{c}$ & $17,41 \mathrm{~d}$ & $77,5 \mathrm{~d}$ & $2118 b$ & 1 \\
\hline BRSMG 800A & $37 \mathrm{f}$ & $90 \mathrm{e}$ & $31,10 \mathrm{e}$ & $6,80 \mathrm{~d}$ & 34,35 a & $11,75 \mathrm{~b}$ & $46,10 \mathrm{~b}$ & $1,87 \mathrm{~d}$ & $76,80 \mathrm{~d}$ & $40,90 \mathrm{~d}$ & $1,50 \mathrm{e}$ & $26,75 \mathrm{~b}$ & $55,07 \mathrm{c}$ & $16,70 \mathrm{e}$ & 20,19 a & $75,0 \mathrm{e}$ & $1989 \mathrm{c}$ & 1 \\
\hline BRS Valiosa RR & $40 \mathrm{c}$ & $99 \mathrm{c}$ & $37,55 \mathrm{~d}$ & $9,85 \mathrm{~b}$ & 34,75 a & $13,42 \mathrm{a}$ & 48,15 a & $2,24 \mathrm{~b}$ & 239,05 a & $105,80 \mathrm{~b}$ & $2,60 \mathrm{~d}$ & $8,50 \mathrm{e}$ & $50,40 \mathrm{c}$ & $38,52 \mathrm{c}$ & $18,29 \mathrm{c}$ & $81,2 \mathrm{~b}$ & $1829 \mathrm{c}$ & 1 \\
\hline TMG 155 RR & $38 \mathrm{e}$ & $99 \mathrm{c}$ & $34,30 \mathrm{~d}$ & $7,80 \mathrm{c}$ & 34,67 a & $13,15 \mathrm{a}$ & $47,82 \mathrm{a}$ & $2,36 \mathrm{~b}$ & $199,65 \mathrm{~b}$ & $83,55 \mathrm{c}$ & $2,12 \mathrm{~d}$ & $12,40 \mathrm{~d}$ & $34,72 \mathrm{e}$ & $50,75 \mathrm{~b}$ & $18,55 \mathrm{c}$ & $73,7 \mathrm{f}$ & $1681 \mathrm{c}$ & 1 \\
\hline M-Soy 7211 & $34 \mathrm{~h}$ & $86 \mathrm{f}$ & $50,00 \mathrm{~b}$ & $6,75 \mathrm{~d}$ & 34,50 a & $13,45 \mathrm{a}$ & $47,92 \mathrm{a}$ & $1,98 \mathrm{~d}$ & $103,70 \mathrm{~d}$ & $51,95 \mathrm{~d}$ & $5,85 \mathrm{~b}$ & $12,45 \mathrm{~d}$ & $58,95 \mathrm{~b}$ & $22,70 \mathrm{~d}$ & $16,76 \mathrm{e}$ & $82,7 \mathrm{a}$ & $1399 \mathrm{c}$ & 1 \\
\hline AH09-001 & $27 \mathrm{i}$ & $86 \mathrm{f}$ & 64,15 a & $6,10 \mathrm{~d}$ & 33,57 a & $11,97 \mathrm{~b}$ & $45,55 \mathrm{~b}$ & $2,22 \mathrm{~b}$ & $175,55 \mathrm{c}$ & $78,80 \mathrm{c}$ & $3,22 \mathrm{~d}$ & $12,52 \mathrm{~d}$ & $43,60 \mathrm{~d}$ & $40,60 \mathrm{c}$ & $13,22 \mathrm{i}$ & $74,0 \mathrm{e}$ & $3286 \mathrm{~b}$ & 2 \\
\hline BRS Luziânia & $28 \mathrm{i}$ & $91 \mathrm{e}$ & $35,50 \mathrm{~d}$ & $7,60 \mathrm{c}$ & $33,30 \mathrm{a}$ & $11,70 \mathrm{~b}$ & $45,00 \mathrm{~b}$ & $1,98 \mathrm{~d}$ & $152,35 \mathrm{c}$ & $76,70 \mathrm{c}$ & $5,85 \mathrm{~b}$ & $20,72 \mathrm{c}$ & $43,82 \mathrm{~d}$ & $29,65 \mathrm{~d}$ & $14,83 \mathrm{~h}$ & $76,5 \mathrm{~d}$ & $3214 b$ & 2 \\
\hline AH09-003 & $28 \mathrm{i}$ & $91 \mathrm{e}$ & $38,10 \mathrm{c}$ & $8,15 \mathrm{c}$ & $34,82 \mathrm{a}$ & $13,35 \mathrm{a}$ & $48,37 \mathrm{a}$ & $2,47 \mathrm{a}$ & $186,57 \mathrm{~b}$ & $75,20 \mathrm{c}$ & $0,75 \mathrm{e}$ & $7,50 \mathrm{e}$ & $35,65 \mathrm{e}$ & $56,12 \mathrm{~b}$ & $16,30 \mathrm{f}$ & $75,0 \mathrm{e}$ & $3079 \mathrm{~b}$ & 2 \\
\hline
\end{tabular}




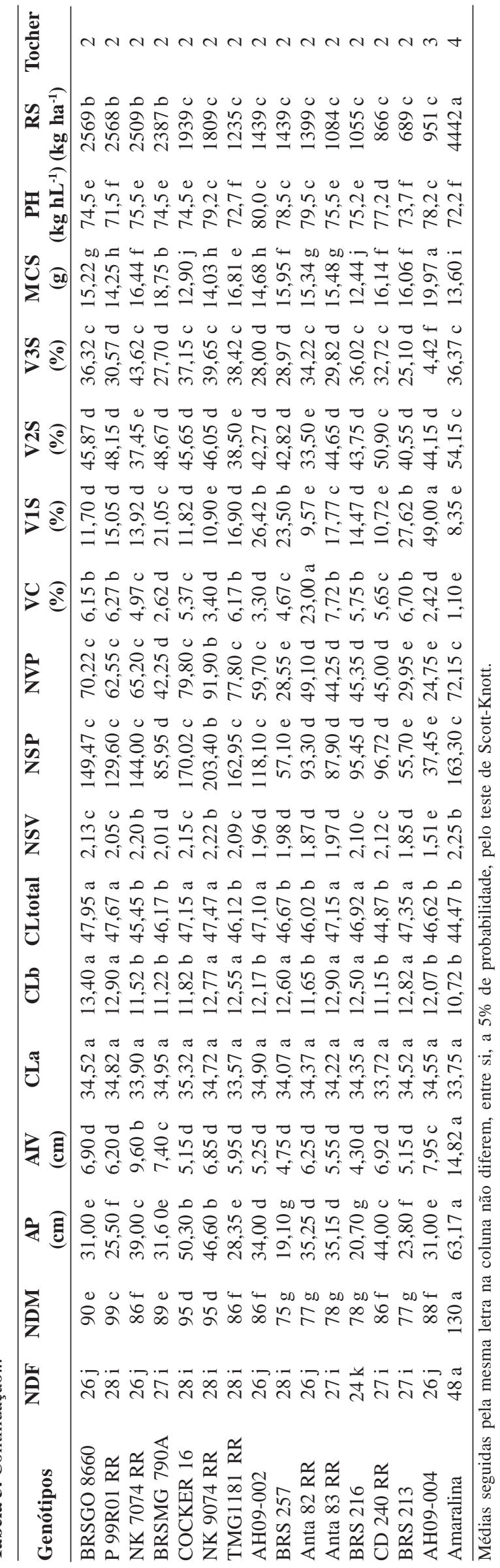

nética com soja, Almeida et al. (2011) também verificaram que o florescimento foi uma das características que mais contribuíram para a dissimilaridade genética encontrada.

$\mathrm{O}$ rendimento de sementes contribuiu com apenas $0,41 \%$ da dissimilaridade (Tabela 4). Entretanto, essa característica é de fundamental importância em estudos de divergência genética, pois a escolha dos progenitores, além da dissimilaridade, deve também considerar o alto rendimento de grãos para a obtenção de progênies superiores (Elias et al., 2007). Essa baixa contribuição relativa para a dissimilaridade genética, em estudos sobre divergência genética, referentes à produtividade de grãos, também foi verificada por Dotto et al. (2010), em milho, e Cabral et al. (2011), em feijão.

A análise de agrupamento dos genótipos pelo método de Tocher utilizou a distância genética entre os pares de genótipos. Esse método tem como princípio manter a homogeneidade dentro e a heterogeneidade entre os grupos e possibilitou a reunião dos 48 genótipos em quatro grupos distintos (Tabela 2). O Grupo I foi composto por 29 (60,4\% dos genótipos); Grupo II foi integrado por 17 (35,4\% dos genótipos); o genótipo AH09-004 integrou o Grupo III e o genótipo Amaralina RR formou o Grupo IV.

O corte no dendrograma gerado pelo método UPGMA foi efetuado a $30 \%$, ponto em que se observou mudança brusca de nível, conforme a recomendação de Cruz (1990). Assim, foram identificados quatro grupos (Figura 1), com características semelhantes às dos grupos obtidos pelo método de Tocher, obedecendo à mesma relação de similaridade entre os genótipos. O genótipo Amaralina (26) (Grupo IV), quando comparado com os 47 demais, foi apon-

Tabela 4. Contribuição relativa das características para a dissimilaridade genética de 48 genótipos de soja, pelo método proposto por Singh (1981), em ordem decrescente de importância, cultivados entressafra de 2010

\begin{tabular}{lc}
\hline Variáveis & Valor $(\%)$ \\
\hline 1. \% vagens com três sementes & 32,62 \\
2.\% de vagens com uma semente & 23,16 \\
3. Número de dias florescimento & 13,42 \\
4. \% de vagens chochas & 8,23 \\
5. Massa de 100 sementes & 4,15 \\
6. Número de vagens por planta & 3,10 \\
7. Número de sementes planta & 2,96 \\
8. Número de semente por vagens & 2,89 \\
9. Número de dias para maturação & 2,13 \\
10. Clorofila total & 1,78 \\
11. Altura de plantas & 1,39 \\
12. Clorofila b & 1,36 \\
13. \% vagens com duas sementes & 1,00 \\
14. Peso de hectolitro & 0,91 \\
15. Rendimento de sementes & 0,41 \\
16. Altura de inserção de vagem & 0,27 \\
17. Clorofila a & 0,13 \\
\hline
\end{tabular}


tado como o mais divergente, como pode ser comprovado no dendrograma pelo método de agrupamento UPGMA (Figura 1).

A menor distância identificada entre todos os pares avaliados foi entre os cultivares Juliana (16) e Juliana RR (18) (Figura 1); esses resultados confirmam seu parentesco, uma vez que o cultivar Juliana é progenitor de Juliana RR. Quanto aos genótipos tipo alimento, a menor distância foi observada entre BRS 257 (8) e BRS 213 (10). Ambos são triplo nulos (isentos dos três genes que codificam as enzimas lipoxigenases) e oriundos do mesmo programa de melhoramento genético (Embrapa Soja).

No estudo realizado por Azevedo et al. (2004), sobre a divergência genética de genótipos de soja com ausência de lipoxigenases, esses autores verificaram que os genótipos triplo nulos (TN) foram inseridos no mesmo grupo e acredita-se que essa similaridade tenha ocorrido pelo fato de esses genótipos possuírem base genética estreita, uma vez que os genótipos TN são oriundos de mutação, e por apresentarem acessos limitados em condições naturais e com poucos cultivares melhorados para esse caráter.

\begin{tabular}{|c|c|c|c|}
\hline 16.7 & VC12 & 0,32 & 99,31 \\
\hline 18. & VC13 & 0,21 & 99,52 \\
\hline $\begin{array}{l}42 \\
45\end{array}$ & VC14 & 0,15 & 99,67 \\
\hline $15-$ & VC15 & 0,12 & 99,80 \\
\hline 33. - & VC16 & 0,12 & 99,92 \\
\hline & VC17 & 0,07 & 100,0 \\
\hline
\end{tabular}

As duas primeiras variáveis canônicas explicaram aproximadamente $80 \%$ da variância total encontrada (Tabela 5). $\mathrm{O}$ que permite explicar satisfatoriamente a variabilidade manifestada entre os genótipos avaliados (Figura 2). No estudo realizado por Krause et al. (2009), verificou-se que as duas primeiras variáveis canônicas explicaram apro-

Tabela 5. Estimativas das variâncias (autovalores), variâncias percentuais acumuladas das variáveis canônicas, visando a estimar a dissimilaridade genética entre 48 genótipos de soja, entressafra 2010

\begin{tabular}{lcc}
\hline $\begin{array}{l}\text { Variáveis } \\
\text { Canônicas }\end{array}$ & $\begin{array}{c}\text { Variâncias } \\
\text { (autovalores) }\end{array}$ & $\begin{array}{c}\text { Variâncias } \\
\text { Acumuladas \% }\end{array}$ \\
\hline VC1 & 59,02 & 59,02 \\
VC2 & 19,08 & 78,10 \\
VC3 & 5,40 & 83,51 \\
VC4 & 4,13 & 87,64 \\
VC5 & 3,17 & 90,82 \\
VC6 & 2,78 & 93,60 \\
VC7 & 2,18 & 95,78 \\
VC8 & 1,29 & 97,08 \\
VC9 & 0,92 & 98,00 \\
VC10 & 0,61 & 98,62 \\
VC11 & 98,98 \\
VC12 & 99,31 \\
VC13 & 0,35 & 99,52 \\
VC14 & 0,32 & 99,67 \\
VC15 & 0,21 & 99,80 \\
VC16 & 0,15 & 99,92 \\
VC17 & 0,12 & 100,0 \\
\hline
\end{tabular}
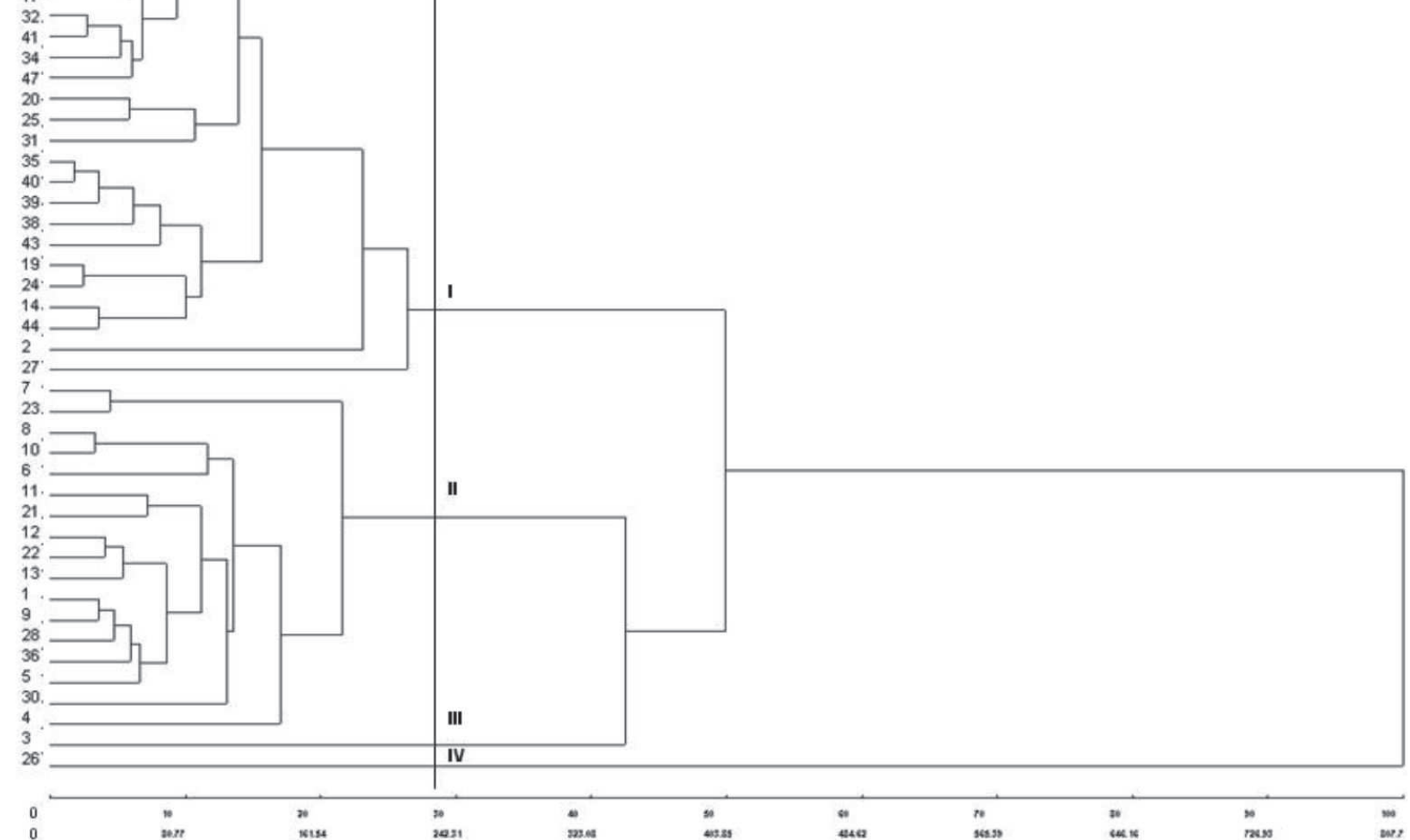

Figura 1. Dendrograma representativo da dissimilaridade genética entre 48 genótipos de soja (Tabela 1), obtidos pelo método de agrupamento UPGMA, utilizando a distância generalizada de Mahalanobis como medida de dissimilaridade, na entressafra de 2010. 


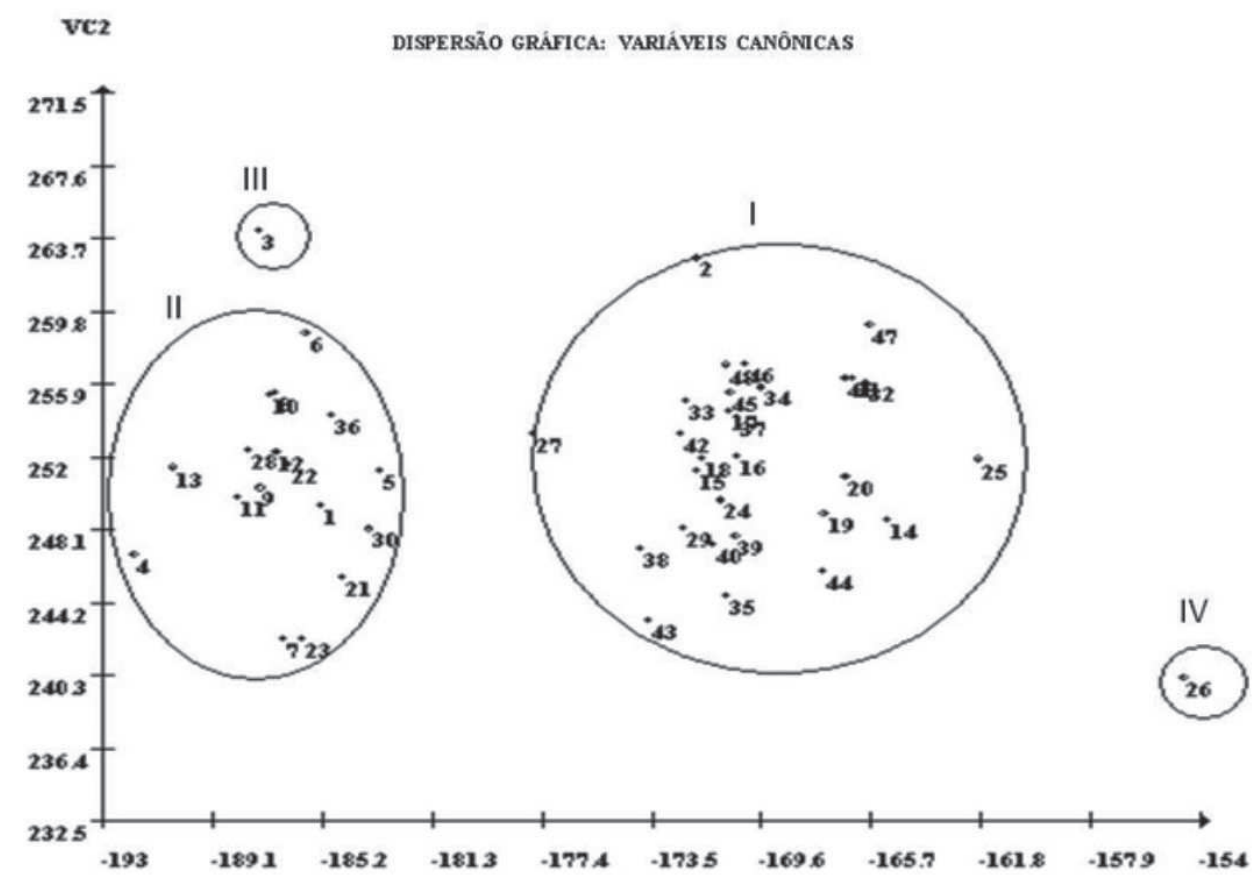

Figura 2. Dispersão gráfica de 48 genótipos de soja (Tabela 1), em relação às duas primeiras variáveis canônicas, estabelecidos pela combinação linear de dezessete características fisiológicas e morfoagronômicas.

ximadamente $80 \%$ da variação total. Condé et al. (2010), no trabalho sobre divergência na cultura do trigo, observaram que as duas primeiras variáveis canônicas explicaram mais de $80 \%$ da variância total.

O padrão de similaridade dos genótipos também pode ser conferido pela dispersão gráfica das variáveis canônicas (Figura 2), em que foram divididos em quatro grupos, concordando com os resultados obtidos pelos métodos de Tocher e UPGMA. Vogt et al. (2010), no trabalho sobre divergência genética com girassol, também verificaram que os métodos de agrupamento de Tocher, da UPGMA e das Variáveis Canônicas foram concordantes entre si.

Para Abreu et al. (2004), essa identificação de genótipos contrastantes por meio das técnicas multivariadas e dos agrupamentos é relevante para que os programas de melhoramento obtenham êxito; entretanto, a escolha de genótipos deve ser feita considerando-se, também, seus comportamentos "per se", ou seja, recomendam-se cruzamentos entre genótipos divergentes, mas que também apresentem desempenho superior em relação às principais características agronômicas.

Diante disso, uma análise de comparação de médias, juntamente com os agrupamentos estabelecidos pelo método de Tocher, da UPGMA e das Variáveis Canônicas, permite a identificação dos cruzamentos promissores para obtenção de cultivares transgressivos.

Sendo assim, poderão ser esperadas como promissoras as seguintes hibridações: M-Soy 8766, M-Soy 9144, A 7002 e M-Soy 9056, do Grupo I, com Amaralina, do Grupo IV, para produção de grãos (destinados a óleo e farelo), por serem dissimilares e apresentarem médias elevadas de rendimento de sementes, e cruzamentos entre os cultivares M-Soy 8766, M-Soy 9144 (Grupo I) e Amaralina (Grupo IV), com BRSMG 790A, BRS 257, BRS 216 e BRS 213 (Grupo III), visando a genótipos de soja especial (destinada à alimentação humana), possivelmente, envolvendo retrocruzamentos, para fixar o caráter de melhor sabor (ausência dos genes que codificam as enzimas lipoxigenases), naqueles cultivares que apresentam insensibilidade fotoperiódica e que apresentam alto rendimento de sementes.

\section{CONCLUSÕES}

Verificou-se divergência genética entre os genótipos de soja, cultivados em várzea irrigada no Estado do Tocantins;

Os métodos de agrupamento de Tocher, da UPGMA e das Variáveis Canônicas são concordantes entre si e formaram quatro grupos distintos;

As seguintes hibridações são promissoras para produção de grãos destinados a óleo e farelo: M-Soy 8766, M-Soy 9144, A 7002 e M-Soy 9056 com Amaralina;

Cruzamentos entre M-Soy 8766, M-Soy 9144 e Amaralina com BRSMG 790A, BRS 257, BRS 216 e BRS 213 são indicados, visando a genótipos de soja especial para alimentação humana. 


\section{AGRADECIMENTOS}

À Coordenação de Aperfeiçoamento de Pessoal de Nível Superior (CAPES), pela concessão da bolsa de estudo durante o curso de Mestrado.

À EMBRAPA Soja e EPAMIG, pelo fornecimento dos cultivares de soja tipo alimento.

\section{REFERÊNCIAS}

Abreu FB, Leal NR, Rodrigues R, Amaral JRAT \& Silva DJH (2004) Divergência genética entre acessos de feijão-de-vagem de crescimento indeterminado. Horticultura Brasileira, 22:547-552.

Almeida RD, Peluzio JM \& Afférri FS (2011) Divergência genética entre cultivares de soja, sob condições de várzea irrigada, no sul do Estado Tocantins. Revista Ciência Agronômica, 42:108115 .

Azevedo PH, Azevedo VH, Sediyama T, Reis MS, Teixeira RC \& Cecon PR (2004) Divergência genética entre genótipos de soja ausentes de enzimas lipoxigenases. Revista Ceres, 51:663-670.

Bellaver C, Cotrefal G \& Grecco M (2002) Soja integral: processamento e uso. Alimento Animal, 7:28-30.

Benin G, Carvalho FI, Assmann IC, Cigolini J, Cruz PJ, Marchioro VV, Lorencetti C \& Silva JAG (2002) Identificação da dissimilaridade genética entre genótipos de feijoeiro comum (Phaseolus vulgaris L.) do grupo preto. Revista Brasileira de Agrociência, 8:179-184.

Cabral PDS, Soares TCBS, Lima ABP, Alves DS \& Nunes JA (2011) Diversidade genética de acessos de feijão comum por caracteres agronômicos. Revista Ciência Agronômica, 42:898-905.

Condé ABT, Coelho MAO, Fronza V \& Souza LV (2010) Divergência genética em trigo de sequeiro por meio de caracteres morfoagronômicos. Revista Ceres, 57:762-767.

Critchley C (1999) Concepts in photobiology: photosinthesis and photomorphogenesis. New Delhi, Narosa Publishing House. $587 \mathrm{p}$.

Cruz CD (2008) Programa Genes: diversidade genética. Viçosa, Editora UFV, 278p.

Cruz, CD (1990) Aplicação de algumas técnicas multivariadas no melhoramento de plantas. Tese de Doutorado. Escola Superior de Agricultura Luiz de Queiroz, Piracicaba, 188p.

Dotto MA, Afférri FS, Peluzio JM, Melo AV \& Carvalho EV (2010) Divergência genética entre cultivares comerciais de milho em baixas altitudes no Tocantins, safra 2007/2008. Revista Ciência Agronômica, 41:630-637.

Duffy C, Perez K \& Partridge A (2007) Implications of phytoestrogen intake for breast cancer. CA Cancer J Clin, 57:260-277.

EMBRAPA. Centro Nacional de Pesquisa de Solos. Sistema brasileiro de classificação de solos (1999). Brasília, Embrapa Serviço de Produção de Informação. 412p.

EMBRAPA. Tecnologias de produção de soja - região central do Brasil 2009 e 2010 (2008). Londrina, Embrapa Soja. Sistemas de Produção, n.13. 262p.

Elias TH, Vidigal MGC, Gonela A \& Vogt GA (2007) Variabilidade genética em germoplasma tradicional de feijão-preto em Santa Catarina. Pesquisa Agropecuária Brasileira, 42:1443-1449.

Kloster GS, Barelli MAA, Silva CR, Neves, LG, Paiva Sobrinho S \& Luz PB (2011) Análise da divergência genética através de caracteres morfológicos em cultivares de feijoeiro. Revista Brasileira de Ciências Agrárias, 6:452-459.
Krause W, Rodrigues R, Gonçalves LSA, Bezerra Neto FV \& Leal NR (2009) Genetic divergence in snap bean based on agronomic traits and resistance to bacterial wilt. Crop Breeding and Applied Biotechnology, 9:246-252.

Marques MC (2010) Adaptabilidade, estabilidade e divergência genética de cultivares de soja em três épocas de semeadura, em Uberlândia, MG. Dissertação de mestrado. Universidade Federal de Viçosa, 84p.

Peluzio JM, Afférri FS, Monteiro FJF, Melo AV \& Pimenta RS (2010) Adaptabilidade e estabilidade de cultivares de soja em várzea irrigada no Tocantins. Revista Ciência Agronômica, 41:427-434

Sediyama T. Tecnologia de produção da soja. Editora Mecenas LTDA (2009). Londrina, 314p.

SEPLAN. Secretaria do Planejamento e Meio Ambiente do Tocantins (2003). Atlas do Tocantins: subsídios ao planejamento da gestão territorial. $3^{\mathrm{a}}$ ed. Palmas, TO: SEPLAN. 49p.

Singh D (1981) The relative importance of characters affecting genetic divergence. The Indian Journal of Genetic and Plant Breeding, 41:237-245.

Vogt GA, Balbinot Júnior AA \& Souza AM (2010) Divergência genética entre cultivares de girassol no Planalto Norte Catarinenses. Scientia Agrária, 11:307-315. 